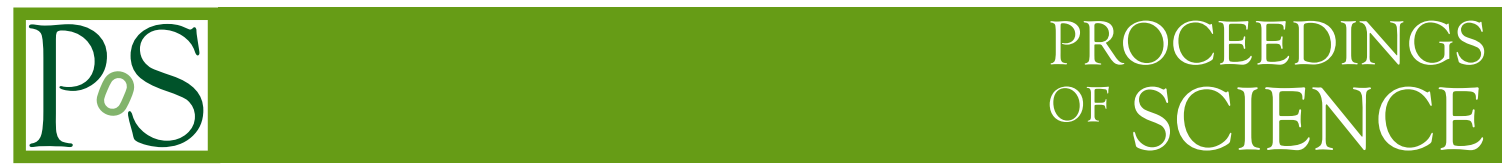

\title{
NGC 4151 with INTEGRAL: reflection on IC emission
}

\author{
Roland Walter* \\ ISDC Data Centre for Astrophysics, Chemin d'Ecogia 16,CH-1290 Versoix, Switzerland \\ Observatoire de Genève, Université de Genève, Chemin des Maillettes 51, 1290 Sauverny, \\ Switzerland \\ E-mail: Roland.Walter@unige.ch
}

\section{Piotr Lubiński}

Centrum Astronomiczne im. M. Kopernika, Bartycka 18, PL-00-716 Warszawa, Poland

E-mail: lubinski@ncac.torun.pl

We present a comprehensive spectral analysis of all INTEGRAL observations of NGC 4151 together with RXTE, XMM, Swift and Suzaku data. The range of plasma and reflection parameters are determined with an unprecedented accuracy. The geometry of the Comptotnisation cloud could be studied. A close and a distant reprocessing region are disentangled. The spectral variability favors a hot inner accretion flow and a cold outer disc with a geometry similar to that inferred for black-hole binaries.

The Extreme sky: Sampling the Universe above $10 \mathrm{keV}$

October 13-17 2009

Otranto (Lecce) Italy

\footnotetext{
* Speaker.
} 


\section{Introduction}

The basic properties of the hard X-ray and soft $\gamma$-ray emission from the radio-quiet Seyfert galaxies nuclei are relatively well known thanks to the several satellites operating during the last two decades. The spectra are commonly approximated by a phenomenological e-folded cut-off power-law model accompanied usually by a Compton reflection component.

The nearby $(\mathrm{z}=0.0033)$ Seyfert galaxy NGC 4151 is the second (after Cen A) brightest, persistent AGN in the $20-100 \mathrm{keV}$ band. Thanks to its brightness and proximity it is extensively studied in all wave bands, with quite well established properties in the radio, infrared, optical, ultraviolet and $\mathrm{X} / \gamma$ bands.

NGC 4151 was observed by INTEGRAL during 8 periods as a primary or secondary target. The results of the first dedicated observation performed in May 2003 were already published (1). The object was found in a spectral state quite similar to those observed with OSSE, however, at the highest flux level ever noticed. There was no evidence for a significant spectral variation, despite the $50 \%$ variations of the absolute flux. The Comptonization model fitted to the summed spectrum resulted in a plasma temperature of $94 \mathrm{keV}$, plasma optical depth about 1.3, reflection component around 0.7 and absorbing $N_{H}$ column density of $\approx 7 \times 10^{22} \mathrm{~cm}^{-2}$. Interestingly, during all later INTEGRAL observations NGC 4151 was catched at a moderately low or very low flux level. This allows us to study the spectral properties of the dim state of this AGN with unprecedented precision and compare them with the very bright state of May 2003.

\section{Data and Analysis}

We used all NGC 4151 data collected so far with INTEGRAL . Good quality data came from the dedicated observations made in May 2003, January 2007, May 2007 and December 2007. The rest of data was taken from observations of the Coma cluster, Mrk 273, NGC 4736, M51 and Mrk 421 , when NGC 4151 was almost always seen at an off-axis angle in the range $9^{\circ}-15^{\circ}$. The ISGRI and SPI data sets comprised all observations made with the source at an off-axis angle smaller than $15^{\circ}$. For JEM-X and OMC the upper off-axis angle for data selection was set to $3^{\circ}$, thus only a selection of data from the dedicated observations were used. The data have been reduced using the Offline Scientific Analysis (OSA) software v. 7.0 provided by the INTEGRAL Science Data Centre, ISDC (2). In general, all OSA pipeline parameters were set to the default values. ISGRI spectra and light curves were extracted with the standard spectral extraction software, applying an input source catalog including: NGC 4151, NGC 4051, NGC 4138, Mrk 766, NGC 4258, NGC 4395, Coma Cluster, NGC 5033, Mrk 421 and Mrk 268. The same catalog was used for SPI spectral extraction. In case of SPI we used several different methods to extract the spectra: spiros with different background modelling options and spimodfit. All these methods gave very similar spectra. JEM-X spectra were prepared from mosaic images stacked for a given observation.

The INTEGRAL dataset was supplemented with all the avalaible X-ray data for NGC 4151 taken since 2003 by the RXTE, XMM-Newton, Swift and Suzaku satellites. These data were used to extract the low-energy spectra, needed to get better constraints on the absorbing column density and the reflection component. Their analysis is described in (3). 


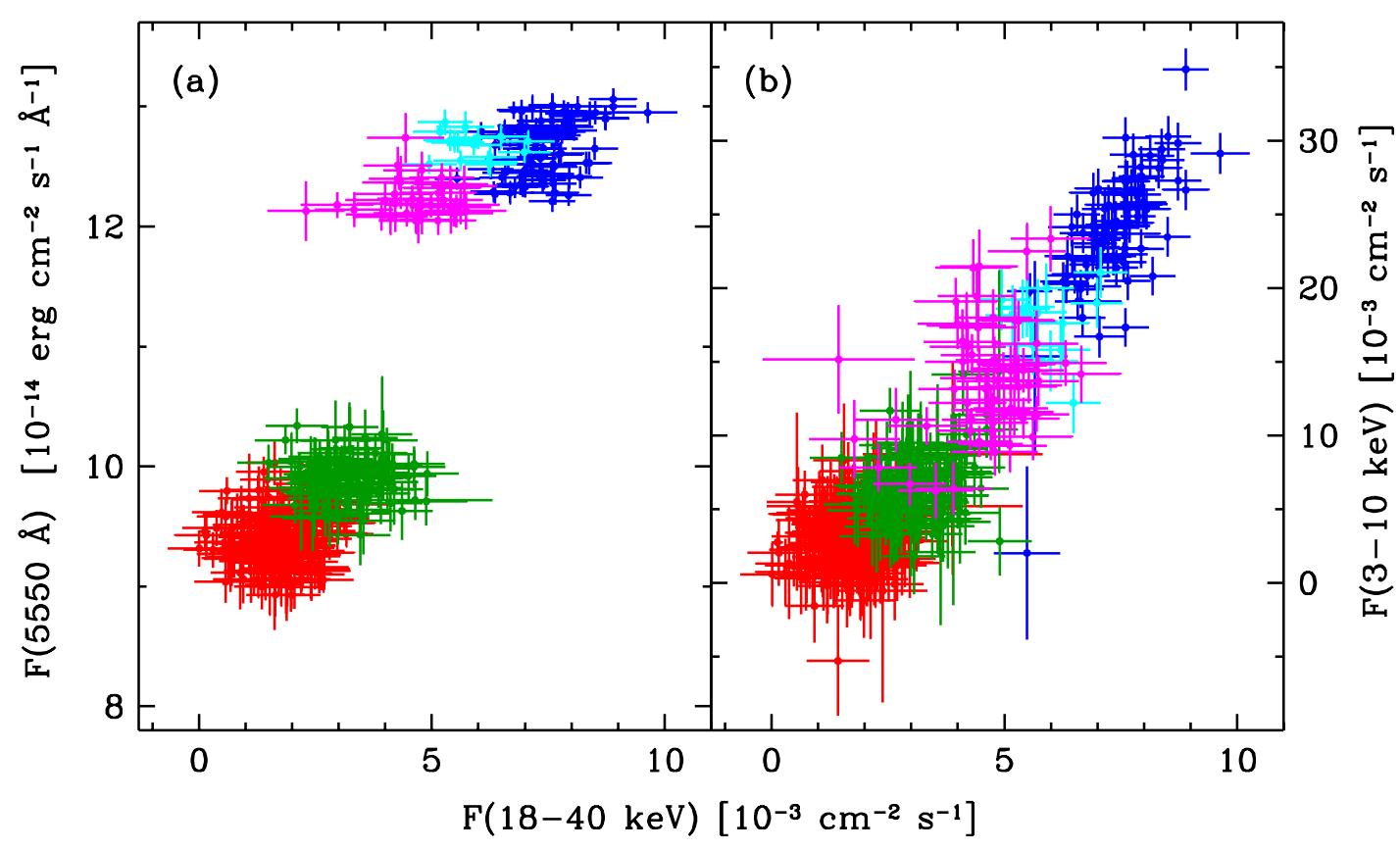

Figure 1: Correlations between the hard X-ray count rate $(18-40 \mathrm{keV})$ and the optical flux at $5550 \AA$ (a) and the soft X-ray (3-10 keV) count rate (b) measured during the main INTEGRAL observations of NGC 4151. Each cross corresponds to a single science window.

\section{Flux correlations}

The INTEGRAL observations are very suitable for correlation studies because there are completely contemporary data in the optical, soft and hard X-ray bands. We used all NGC $4151 \mathrm{ob}-$ servations when the source was within $3^{\circ}$ from the centre of INTEGRAL field of view to study the correlations on the pointing-time scale. Figure 1 shows the correlations between the 18-40 $\mathrm{keV}$ flux and the fluxes observed in the 3-10 keV and in the $\mathrm{V}$ band. Both optical and soft X-ray emission are strongly correlated with the hard X-ray emission, however, the correlation patterns are quite different. Optical data group in two well separated regions, corresponding to the high and low level of optical/hard X-ray fluxes. The 3-10 keV and 18-40 keV fluxes exhibit a single, linear correlation. Statistical uncertainty does not allow to see the correlation for medium and low flux states but during the bright state there is a clear correlation along the general trend line. These results indicate that during all INTEGRAL observations the slope of the X-ray spectrum of NGC 4151 below $40 \mathrm{keV}$ remained constant, with only a slight hardening during the dim state.

Rank-order correlation tests confirm the above observations. A significant probability (> 0.999) of the correlation for ISGRI/OMC data is found only for the bright state and for data sets comprising the high flux states (bright and medium), low flux states (faint and dim) and entire data set. The same happenes for ISGRI/JEM-X data but with a much higher significance.

\section{Spectral analysis}

We defined four dominant spectral states for which significant spectral sets could be obtained 
Table 1: Results of spectral fitting obtained for the two extreme flux states with the compPS continuum model assuming a spherical geometry. Comptonization model parameters: $k T_{e}$ - electron plasma temperature in $\mathrm{keV} ; y$-Compton parameter; $\tau$ - plasma optical depth; $R$ - relative amplitude of the reflection component; $A$ - absolute normalization.

\begin{tabular}{lcccccc}
\hline State & $k T_{e}$ & $y$ & $\tau$ & $R$ & $A$ & $\chi^{2} /$ d.o.f. \\
\hline Bright & $58_{-7}^{+10}$ & $1.06_{-0.02}^{+0.02}$ & $2.3_{-0.4}^{+0.3}$ & $0.39_{-0.05}^{+0.05}$ & $6.4_{-0.7}^{+1.5}$ & $3256.9 / 3472$ \\
Dim & $163_{-11}^{+13}$ & $1.00_{-0.01}^{+0.01}$ & $0.79_{-0.06}^{+0.05}$ & $0.72_{-0.03}^{+0.03}$ & $1.55_{-0.01}^{+0.01}$ & $3385.0 / 3437$ \\
\hline
\end{tabular}

and be fitted with a Comptonization and reflection model assuming that the plasma physical state is similar for periods with a similar hard X-ray flux. We tested that the spectral shape does not vary too much within a given spectral set using ISGRI spectra extracted for single revolutions. The spectral states are the following:

- Dim state: the ISGRI spectrum was extracted from all data from Revs. 0310-0563. The range of relative NGC $415120-100 \mathrm{keV}$ fluxes of these data is $0-25 \%$. Lower energy band was covered by spectra of RXTE PCA PCU2, XMM-Newton EPIC PN and Suzaku XIS. Supplementary spectra of the entire set were: two INTEGRAL JEM-X 1 spectra (Revs. 0521-0522 and Revs. 0561-0563), Suzaku PIN, RXTE HEXTE (cluster 1) and INTEGRAL SPI spectrum summed for the same period as ISGRI.

- Faint state: ISGRI data from Revs. 0634, 0636, 0678 and 0679 were merged into one spectrum. Corresponding 20-100 keV relative fluxes are within 15-45\%. Because there were no RXTE observations close in time, the medium energy range data for the spectral fitting were supplied by INTEGRAL JEM-X 1 spectrum from Revs. 0634 and 0636. The main low-energy spectrum of this set consists of a spectrum of XMM-Newton EPIC PN. We used also four Swift XRT spectra and INTEGRAL SPI spectrum, the latter summed over the same period as ISGRI.

- Medium state: both ISGRI and JEM-X spectra comprise data from Revs. 0809-0811. The JEM-X exposure time is much shorter than the ISGRI exposure time because this observation was made in a standard dithering mode, with NGC 4151 often outside the JEM-X field of view. Relative fluxes are in the $24-68 \%$ range.

- Bright state: data from the observation made in May 2003 constitute the bright state set. The 20-100 keV flux of the bright state varied within $64-100 \%$ of the $20-100 \mathrm{keV}$ flux limits of NGC 4151. The spectral set consisted of the ISGRI spectrum, two XMM-Newton EPIC PN spectra and summed RXTE PCA PCU2 spectrum. Complementary spectra are the summed spectrum of INTEGRAL/JEMX-2, RXTE HEXTE cluster 0 and 1 spectra and the INTEGRAL/SPI spectrum.

To determine the physical parameters of the plasma residing in the nucleus of NGC 4151 we used the most advanced standard XSPEC model for Comptonization, namely compps (4), including reflection. The full model in the Xspec notation is: constant*wabs*absori*zpcfabs(compPS+zgauss) 
The resulting best fits are displayed on figure 2, where a spherical geometry is assumed with main parameters listed in table 1. Other geometries and parameters are explored in (3).

\section{Reflection}

The geometry of the reprocessing media in AGN is an important issue for modelling the physics of the central source emitting hard $\mathrm{X}$ and soft $\gamma$ rays. One of the parameters constraining that geometry is the extent of the reflector as seen from the plasma region. The other interesting quantity is the distance between the source and reflector. Because both accretion disc and molecular torus are present in AGN, the reflection strength fitted to a given spectrum is normally a mixture of reflection characterizing both these media. Thanks to the quite precise measurements of the reflection strength for the bright and dim state of NGC 4151, we can disentangle two reflection subcomponents (under some simplifying assumptions). Using the fluxes determined from the fitted models we obtained values of $R_{\text {Torus }}=0.31 / 2 \pi$ and $R_{\text {Disk }}=0.24 / 2 \pi$ (see (3) for the details). A comparison of the reflection and Iron line strengths suggest a two phase accretion disc.

\section{Hard X-ray source}

Owing to unprecedented quality and amount of spectral data we were able to determine with a high precision several parameters of two extreme hard X-ray flux states of NGC 4151 (table 1). Our

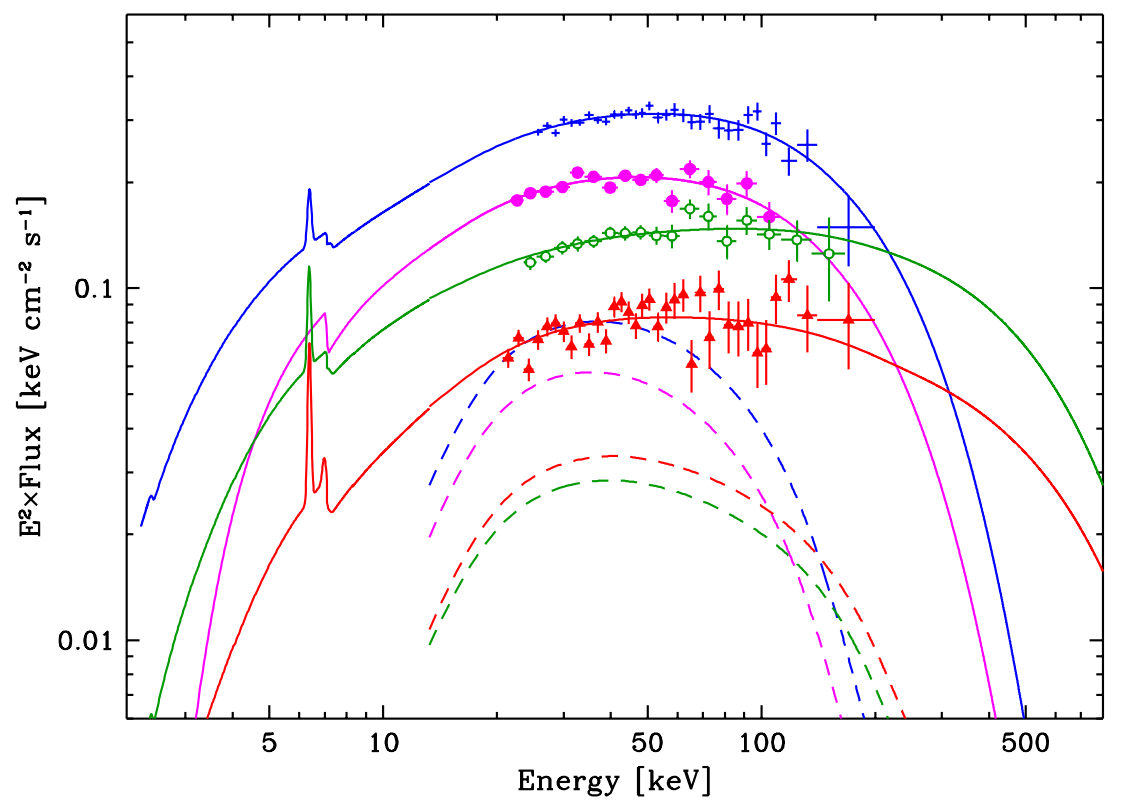

Figure 2: Best fit spectra for the four hard X-ray flux states, derived from observations with INTEGRAL and other satellites. Only ISGRI data are presented for clarity: bright state - crosses, medium state - dots, faint state - circles, dim state - triangles. Solid lines show the Comptonization models for spherical geometry fitted to the selected spectral sets. Dashed curves below show the reflection components of the model for the bright, medium, dim and faint state, starting from the top. 


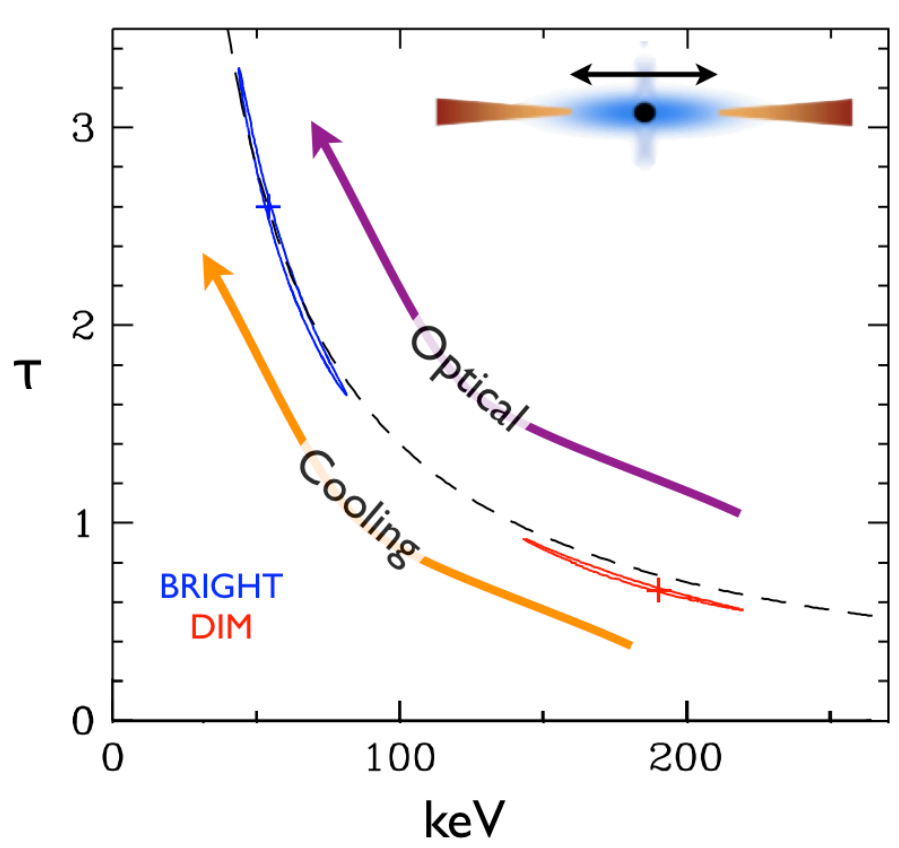

Figure 3: The $90 \%$ confidence regions computed for the plasma temperature and optical depth parameter of the Comptonization models fitted to the bright (blue) and dim (red) state spectra of NGC 4151 .

basic assumption that the spectrum of the object is closely related to the hard X-ray flux appears to be confirmed by the ISGRI and JEM-X flux correlation shown in Fig. 1.

In the bright state, the optical flux is larger, the electron temperature is three times smaller and the Compton optical depth is much larger (Fig. 3). This behavior could be explained with a two phase accretion disk where the inner disk shrink towards a denser corona during the bright state, increasing the electron cooling and lowering the coronal temperature. This geometry is similar to that inferred for black-hole binaries.

\section{References}

[1] Beckmann, V. et al., ApJ 634 (2005) 939.

[2] Courvoisier, T. J.-L. et al., A\&A 411 (2003) L53.

[3] Lubiński, P. et al., MNRAS (2010) in preparation.

[4] Poutanen, J. and Svensson, R., ApJ 470 (1996) 249. 